\title{
Evaluation of the role of ischemia modified albumin in neonatal hypoxic-ischemic encephalopathy
}

Mohamed A. Talat, MD¹, Rabab M. Saleh, MD ${ }^{1}$, Mohammed M. Shehab, MD ${ }^{1}$, Naglaa A. Khalifa, MD², Maha Mahmoud Hamed Sakr ${ }^{2}$, Walaa M. Elmesalamy ${ }^{3}$

Departments of ${ }^{1}$ Pediatrics and ${ }^{2}$ Clinical Pathology, Faculty of Medicine, Zagazig University, Zagazig, Egypt; ${ }^{3}$ Ministry of Health, Zagazig, Egypt

Background: Birth asphyxia is a leading cause of neonatal mortality. Ischemia-modified albumin (IMA) levels may have a predictive role in the identification and prevention of hypoxic disorders, as they increase in cases of ischemia of the liver, heart, brain, bowel, and kidney.

Purpose: This study aimed to assess the value of IMA levels as a diagnostic marker for neonatal hypoxic-ischemic encephalopathy (HIE).

Methods: Sixty newborns who fulfilled 3 or more of the clinical and biochemical criteria and developed HIE as defined by Levene staging were included in our study as the asphyxia group. Neonates with congenital malformation, systemic in fection, intrauterine growth retardation, low-birth weight, cardiac or hemolytic disease, family history of neurological diseases, congenital or perinatal infections, preeclampsia, diabetes, and renal diseases were excluded from the study. Sixty healthy neonates matched for gestational age and with no maternal history of illness, established respiration at birth, and an Apgar score $\geq 7$ at 1 and 5 minutes were included as the control group. IMA was determined by double-antibody enzymelinked immunosorbent assay of a cord blood sample collected within 30 minutes after birth.

Results: Cord blood IMA levels were higher in asphyxiated newborns than in controls $(250.83 \pm 36.07 \mathrm{pmol} / \mathrm{mL}$ vs. $120.24 \pm 38.9 \mathrm{pmol} / \mathrm{mL}$ ). Comparison of IMA levels by HIE stage revealed a highly significant difference among them $(207.3 \pm 26.65,259.28 \pm 11.68,294.99 \pm 4.41 \mathrm{pmol} / \mathrm{mL}$ for mild, moderate, and severe, respectively). At a cutoff of 197.6 $\mathrm{pmol} / \mathrm{mL}$, the sensitivity was $84.5 \%$, specificity was $86 \%$, positive predictive value was $82.8 \%$, negative predictive value was $88.3 \%$, and area under the curve was $0.963(P<0.001)$.

Conclusion: IMA levels can be a reliable marker for the early diagnosis of neonatal HIE and can be a predictor of injury severity.

Key words: Ischemia modified albumin, Hypoxic-ischemic encephalopathy

\section{Key message}

Questions: What is the value of ischemia modified albumin (IMA) as a diagnostic marker for neonatal hypoxic-ischemic encephalopathy (HIE)?

Finding: IMA levels were significantly higher (nearly double elevation) in hypoxic than healthy newborns in the first few hours after birth in the full-term neonates.

Meaning: IMA can be a reliable marker for the early diagnosis of neonatal HIE and can be a predictor of injury severity.

\section{Introduction}

Birth asphyxia is the second leading cause of neonatal mortality after sepsis. Perinatal asphyxia is an insult to the fetus due to oxygen insufficiency (hypoxia) and/or reduction of perfusion (ischemia) to various organs. It is associated with tissue lactic acidosis and hypercapnia. It is the most significant cause of cerebral damage and long-term neurological consequences in the perinatal period both in term and preterm infants. ${ }^{1)}$ Its incidence is approximately 1 to 6 every 1,000 live term births in developing countries, $20 \%-50 \%$ of asphyxiated newborns die within the neonatal period. Up to $25 \%$ of the survivors will display permanent neuropsychological handicaps, including cerebral palsy, mental retardation, epilepsy, or learning disability.2) Several biochemical predictors of hypoxic brain damage have been researched in the last decade such as activin A, creatine kinase, neuron-specific enolase, and protein S-100. ${ }^{3)}$

Ischemia modified albumin (IMA) is a modification of human serum albumin (HSA). hypoxia, acidosis, or ischemia alter the $\mathrm{N}$-terminal amino acids of HAS and decrease the binding capacity of HSA to metals such as cobalt, nickel, and copper. ${ }^{4)}$ It is known that ischemia of liver, heart, brain, bowel, and kidney raise the IMA level. IMA rapidly elevated within 5 to 10 minutes after the ischemic event and stays high for 30 minutes then it returns to baseline 12 hours after the ischemia event. ${ }^{5)}$ Therefore, it may has a predictive value in the identification and

Corresponding author: Mohamed Ahmed Talat Kamal, MD. Department of Pediatrics, Faculty of Medicine, Zagazig University, 3 El-Sayed Eleem St, Manshyyet Abaza, Zagazig, Sharkia, Egypt 
Table 1. Neonatal studies related to the ischemia-modified albumin and hypoxia

\begin{tabular}{|c|c|c|c|c|c|c|c|}
\hline Study & Group & No. & Biological sample & Time of sample collection & Mean IMA level & Unit of assay & $P$ value \\
\hline \multirow[t]{2}{*}{ Gugliucci et al. ${ }^{32)}$} & ND & 12 & Cord blood & Delivery & $0.64 \pm 0.24$ & Absorbance values at $470 \mathrm{~nm}$ & 0.037 \\
\hline & $C D$ & 14 & & & $0.96 \pm 0.5$ & & \\
\hline \multirow[t]{2}{*}{ lacovidou et al. ${ }^{34)}$} & AGA & 110 & Cord blood & Delivery & 112.44 & $\mathrm{U} / \mathrm{mL}$ & $>0.05$ \\
\hline & IUGR & 57 & & & 115.54 & & \\
\hline \multirow[t]{2}{*}{ Kumral et al. ${ }^{35)}$} & ND & 153 & Cord blood & Delivery & 153.3 & $\mathrm{pmol} / \mathrm{mL}$ & 0.015 \\
\hline & $\mathrm{CD}$ & 16 & & & 332.0 & & \\
\hline \multirow[t]{2}{*}{ Biswas et al. ${ }^{17)}$} & ND & 40 & Cord blood & Delivery & 32.44 & $\mathrm{U} / \mathrm{mL}$ & $<0.01$ \\
\hline & $C D$ & 40 & & & 55.7 & & \\
\hline \multirow[t]{2}{*}{ Kar et al. ${ }^{33)}$} & NHP & 40 & Cord blood & Delivery & 31.55 & $\mathrm{U} / \mathrm{mL}$ & $<0.0001$ \\
\hline & NPE & 45 & & & 45.66 & & \\
\hline
\end{tabular}

IMA, ischemia modified albumin; ND, noncomplicated deliveries; CD, complicated deliveries; AGA, appropriate for gestational age; IUGR, intrauterine growth retardation; NHP, newborn of healthy pregnant women; NCP, newborn of complicated pregnancy; NPE, newborn of pre-eclamptic pregnant.

Boldface indicates a statistically significant difference with $P<0.05$.

prevention of birth asphyxia as ischemia of these organs may be seen. ${ }^{6}$ Previous studies about IMA levels and hypoxia have been published (Table 1). Also, some clinical studies have recently indicated elevated levels of IMA in neonatal sepsis, preterm infants born to mothers with preeclampsia, preterm infants with respiratory distress syndrome, infants of diabetic mother, and ischemic conditions such as necrotizing enterocolitis in newborns. ${ }^{7-9)}$

This study aimed to assess the value of IMA as a diagnostic marker for neonatal hypoxic-ischemic encephalopathy (HIE).

\section{Methods}

\section{Study sample}

This prospective study was performed at pediatric department of Zagazig University Hospital in the period between September 2017 and September 2018. This case-control study was approved by the Institutional Ethical Committee of Zagazig University (approval number: ZU-IRB\# 2510-5-7-2017) and in accordance with the Helsinki declaration after a full explanation of the purpose and nature of all procedures used. Written informed consent was obtained from the parents of the patients involved in the study. Further information and documentation to support this are available to on request.

We studied 60 full-term consecutive asphyxiated neonates who delivered at the obstetric department of Zagazig University Hospital due to nonreassuring fetal status (bradycardia, late deceleration of the fetal heart rate, repetitive variable deceleration of the fetal heart rate, and reduced beat-to-beat variability). Asphyxia was defined according to an Apgar score less than 3 at the 5 minutes, $\mathrm{pH}$ less than 7.0 , or base excess less than 12 in cord blood or venous blood taken from newborns within 60 minutes of birth or the need for positive pressure ventilation (more than 3 minutes) or the presence of postnatal clinical complications attributed to hypoxia and/or ischemia such as shock, altered tone, depressed level of consciousness and convulsion. ${ }^{10)}$

We included the neonates who fulfilled 3 or more of the clinical and biochemical criteria and developed HIE, as defined by Levene staging and born between September 2017 to Sep. tember 2018 (asphyxia group). ${ }^{11)}$ An HIE diagnosis based on the Finer classification ${ }^{12)}$ cannot diagnose the disease until 48 hours after the occurrence of the hypoxic/ischemic events has occurred. Sixty healthy neonates with matched gestational age, without a history of maternal illness, established respiration at birth and Apgar score $\geq 7$ at 1 and 5 minutes were included as (a control group).

Infants with congenital malformation, cardiac or hematological diseases, sepsis, intrauterine growth retardation, low-birth weight were excluded from the study. Other exclusion criteria were familial history of neurological diseases, perinatal infections, multiple pregnancies, preeclampsia, and maternal diabetes or renal diseases to remove the effect of these factors on the oxidative stress.

All studied neonates exposed to full history taking including maternal and delivery history with focus on perinatal asphyxia, mode of delivery, gestational age, neonatal Apgar score of at 1 and 5 minutes and type of resuscitation used: oxygen with face mask, a self-inflating bag with positive pressure, cardiac massage and medications for staging the severity of the asphyxiated group.

Clinical assessment included general examination, gestational age estimation using new Ballard score, ${ }^{13)}$ body weight, head circumference, vital signs, and neurological examination within the first 24 hours after birth and for 7 days to classify HIE according to the criteria of Sarnat and Sarnat. ${ }^{14)}$ The alertness, posture, activity, tone, neonatal reflexes, seizures, the number of anticonvulsant drugs were assessed.

Laboratory investigations including: complete blood count was done using Cell-Dyn_1700 (Abbot Diagnostics, Abbott Park, IL, USA), blood gases, C-reactive protein (CRP) was determined using the Tina-Quant C-Reactive protein Gen 3 assays (Roche Diagnostics, Indianapolis, IN, USA) on Roche Modular P800 system. Cord blood samples were collected as early as possible immediately after birth and analyzed for arterial blood gases, $\mathrm{pH}$ determination with a calculation of base excess was 
analyzed on Roche OMNI.C (Roche Diagnostics GmbH, Mannheim, Germany).

IMA assay, $5 \mathrm{~mL}$ of cord blood was collected, kept in ice-filled containers and brought to the clinical pathology laboratory of Zagazig University Hospital within 30 minutes hours then serum is separated and stored at $-20^{\circ} \mathrm{C}$ for IMA estimation. IMA levels were detected in patients and control groups by double-antibody sandwich enzyme-linked immunosorbent assay (ELISA) using a commercially available ELISA (Cat. No: 201-12-1137; Sun Red Biotechnology, Shanghai, China).

\section{Statistical analysis}

Data were checked, entered, and statistically analyzed by IBM SPSS Statistics ver. 19.0 (IBM Co., Armonk, NY, USA). Data were expressed as a mean \pm standard deviation for quantitative variables or number and percentage for categorical variables.

Student $t$ test for continuous variables and chi-square test for categorical variables were used to compare mean values between the 2 independent groups. Data were compared using analysis of variance test for more than 2 groups. For nonparametric data, Mann-Whitney $U$ test was used to compare quantitative variables between the 2 groups and Kruskal-Wallis for more than 2 groups. Correlations between continuous variables were calculated by Pearson coefficient. IMA's role in predicting HIE was calculated using:

Sensitivity (percent of positives detected correctly identified) $=$ true positives/(true positive + false negative)

Specificity (percent of negatives detected correctly identified) $=$ true negatives/(true negative + false positives)

$\mathrm{PV}+=$ true positive/(true positive + false positive), $\mathrm{PV}-=$ true negatives/(true negatives + false negatives). The area under the curve (AUC) was derived from the receiver operating characteristic curve.

$P$ value of $>0.05$ was insignificant, $P<0.05$ was significant and $P<0.001$ was highly significant.

\section{Results}

Our study included 60 patients with asphyxia (male:female, $36: 24)$, delivered by elective cesarean section $(n=10)$ or vaginally $(n=50)$ and 60 healthy subjects as a control group (male: female, 21:39), delivered by elective cesarean section $(n=15)$ or vaginally $(n=45)$, the history, demographic data and laboratory investigations of the study population are shown in Table 2. The cord blood serum IMA levels were also higher in newborns with perinatal asphyxia than the healthy controls with $P<0.001$.

There was no statistically significant difference between asphyxiated and control groups regarding the mode of delivery as shown in Table 2. In both groups, the mean cord blood IMA levels were slightly elevated in neonates from cesarean sections compared to those from vaginal deliveries but it was not statistically significant as shown in Table 3.

According to the criteria of Sarnat and Sarnat, we classify the asphyxiated group into 3 stages and the percentages of the 3 stages are (stage I, 30\%; stage II, 50\%; and stage III, 20\%). The history, demographic data, and laboratory investigations of the 3 stages are shown in Table 4.

There was a statistically significant difference between asphyxiated and control groups regarding the mortality, where 12 cases $(20 \%)$ were dead in the asphyxiated group in comparison to zero among the control group as shown in Table 2. The percentage of mortality at different stages of HIE are $0 \%, 13.3 \%$, $67.7 \%$ in stage I, II, III respectively.

The cord blood IMA levels were also higher in the dead newborns with perinatal asphyxia as compared to survived newborns with perinatal asphyxia (293.22 $\pm 7.65 \mathrm{pmol} / \mathrm{mL}$ vs. $240.23 \pm 32.21 \mathrm{pmol} / \mathrm{mL}$, respectively with $P=0.001)$. Also, there was a highly significant difference between the 3 stages of

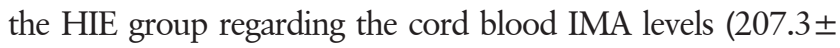
$26.65,259.28 \pm 11.68,294.99 \pm 4.41$ in mild, moderate, severe stages, respectively) as shown in Table 4.

The degree of correlation between the different parameters of the cases and controls are shown in Table 5. In this study, at a cutoff level of IMA $197.6 \mathrm{pmol} / \mathrm{mL}$, the sensitivity was $84.5 \%$, the specificity was $86 \%$, positive predictive value (PPV) was

Table 2. Comparison of patient history, demographic data and laboratory results

\begin{tabular}{lccc}
\hline Variables & $\begin{array}{c}\text { Asphyxiated } \\
(\mathrm{n}=60)\end{array}$ & $\begin{array}{c}\text { Control } \\
(\mathrm{n}=60)\end{array}$ & $P$ value \\
\hline Sex & & & \\
$\quad$ Male & $36(60)$ & $21(35)$ & 0.12 \\
Female & $24(40)$ & $39(65)$ & \\
Mode of delivery & & & \\
Vaginal & $50(83.3)$ & $45(75)$ & 0.1 \\
Cesarean & $10(16.7)$ & $15(25)$ & \\
Mortality & & & \\
Survived & $48(80)$ & $60(100)$ & 0.035 \\
Dead & $12(20)$ & $0(0)$ & \\
Gestational age (wk) & $40.15 \pm 1.18$ & $39.8 \pm 0.69$ & $<0.15$ \\
Birth weight (kg) & $3.64 \pm 0.38$ & $3.10 \pm 0.21$ & $<0.001$ \\
Apgar score, 1 min & $2.40 \pm 0.50$ & $7.35 \pm 0.98$ & $<0.001$ \\
Apgar score, 5 min & $4.55 \pm 0.88$ & $8.7 \pm 1.16$ & $<0.001$ \\
pH & $7.22 \pm 0.11$ & $7.38 \pm 0.03$ & $<0.001$ \\
PO2 (mmHg) & $76.16 \pm 12.64$ & $94.85 \pm 9.20$ & $<0.001$ \\
PCO2 (mmHg) & $47.72 \pm 8.05$ & $39.3 \pm 3.61$ & $<0.001$ \\
Hb (g/dL) & $14.82 \pm 1.83$ & $16.05 \pm 1.87$ & 0.032 \\
IMA (pmol/mL) & $250.83 \pm 36.07$ & $120.24 \pm 38.9$ & $<0.001$ \\
CRP (mg/L) & $4.80 \pm 2.90$ & $3.0 \pm 1.9$ & 0.002 \\
\hline Va & & & \\
\hline
\end{tabular}

Values are presented as number (\%) or mean \pm standard deviation. IMA, ischemia modified albumin; $\mathrm{Hb}$, hemoglobin; CRP, C-reactive protein. Boldface indicates a statistically significant difference with $P<0.05$.

Table 3. Ischemia-modified albumin levels by mode of delivery

\begin{tabular}{lccc}
\hline Variable & Vaginal & Cesarean & $P$ value \\
\hline Asphyxiated $(n=60)$ & $238.53 \pm 34.60$ & $254.53 \pm 35.40$ & 0.13 \\
Control $(n=60)$ & $154.86 \pm 10.85$ & $178.45 \pm 20.30$ & 0.105 \\
\hline
\end{tabular}

Values are presented as mean \pm standard deviation. 
Table 4. Comparison of different stages of hypoxic-ischemic encephalopathy in terms of history, demographic data, and laboratory results

\begin{tabular}{lcccc}
\hline Variable & $\begin{array}{c}\text { I (mild) } \\
(\mathrm{n}=18)\end{array}$ & $\begin{array}{c}\| \text { (moderate) } \\
(\mathrm{n}=30)\end{array}$ & $\begin{array}{c}\| \text { (severe) } \\
(\mathrm{n}=12)\end{array}$ & $P$ value \\
\hline Gestational age $(\mathrm{yr})$ & $39.66 \pm 0.81$ & $40.3 \pm 1.05$ & $40.1 \pm 1.7$ & 0.239 \\
Apgar, 1 min & $2.83 \pm 0.40$ & $2.30 \pm 48$ & $2.0 \pm .0$ & 0.015 \\
Apgar, 5 min & $5.83 \pm 0.4$ & $4.9 \pm 0.56$ & $2.75 \pm 0.5$ & 0.001 \\
Hospital stay (day) & $12.3 \pm 4.17$ & $25.0 \pm 7.9$ & $37.25 \pm 6.18$ & $<0.001$ \\
$\mathrm{pH}$ & $7.29 \pm 0.09$ & $7.22 \pm 0.10$ & $7.11 \pm .11$ & 0.044 \\
$\mathrm{PO} 2$ & $83.08 \pm 9.87$ & $77.08 \pm 13.06$ & $63.5 \pm 5.01$ & 0.043 \\
$\mathrm{PCO} 2$ & $41.83 \pm 8.68$ & $49.35 \pm 5.80$ & $52.47 \pm 8.70$ & 0.074 \\
$\mathrm{Hb}(\mathrm{g} / \mathrm{dL})$ & $14.66 \pm 1.5$ & $14.22 \pm 1.17$ & $16.55 \pm 2.79$ & 0.089 \\
$\mathrm{IMA}(\mathrm{pmol} / \mathrm{mL})$ & $207.3 \pm 26.65$ & $259.28 \pm 11.68$ & $294.99 \pm 4.41$ & $<0.001$ \\
$\mathrm{CRP}(\mathrm{mg} / \mathrm{L})$ & $4.0 \pm 3.09$ & $5.4 \pm 3.2$ & $4.5 \pm 3.7$ & 0.84 \\
\hline
\end{tabular}

Values are presented as mean \pm standard deviation.

$\mathrm{Hb}$, hemoglobin; IMA, ischemia modified albumin; CRP, C-reactive protein. Boldface indicates a statistically significant difference with $P<0.05$.

Table 5. Correlation between ischemia-modified albumin levels and gestational age, birth weight, Apgar score, laboratory results, and arterial blood gas levels of the 2 studied groups

\begin{tabular}{lcccc}
\hline \multirow{2}{*}{ MA } & \multicolumn{2}{c}{ Asphyxia $(\mathrm{n}=60)$} & \multicolumn{2}{c}{ Control $(\mathrm{n}=60)$} \\
\cline { 2 - 5 } & \multicolumn{1}{c}{$r$} & $P$ value & $r$ & $P$ value \\
\hline Gestational age & 0.326 & 0.21 & -0.407 & 0.075 \\
Birth weight & 0.582 & $<0.001$ & -0.349 & 0.132 \\
Apgar score, 1 min & -0.831 & $<0.001$ & 0.118 & 0.621 \\
Apgar score, 5 min & -0.831 & $<0.001$ & 0.117 & 0.622 \\
Hospital stay & 0.834 & $<0.001$ & - & - \\
Hb & 0.164 & 0.312 & 0.284 & 0.226 \\
CRP & 0.449 & $\mathbf{0 . 0 0 4}$ & 0.130 & 0.586 \\
PH & -0.720 & $<0.001$ & -0.259 & 0.270 \\
PO2 & -0.641 & $<0.001$ & 0.002 & 0.994 \\
PCO2 & 0.581 & $<0.001$ & -0.003 & 0.990 \\
\hline
\end{tabular}

IMA, ischemia modified albumin; $\mathrm{Hb}$, hemoglobin; CRP, C-reactive protein. Boldface indicates a statistically significant difference with $P<0.05$.

$82.8 \%$, negative predictive value (NPV) was $88.3 \%$, and the AUC was 0.963 (95\% confidence interval, 0.913-0.99; $P<$ 0.001) as shown in Fig. 1.

\section{Discussion}

Perinatal asphyxia is one of the most critical birth complications and a leading cause of permanent disability and death in full-term neonates.

Our study revealed a significant difference between the 2 studied groups regarding the birth weight and this was attributed to increasing incidence of obstructed labor and hypoxic insult in high birth weight neonates as well as large for gestational age which is matched with other studies which found a strong association of perinatal asphyxia with prolonged labor due to macrosomia. ${ }^{15)}$ On the other hand, our results disagreed with Lee et al. ${ }^{16)}$ who found that lower birth weight in cases of HIE than the control group due to respiratory distress caused by Respiratory

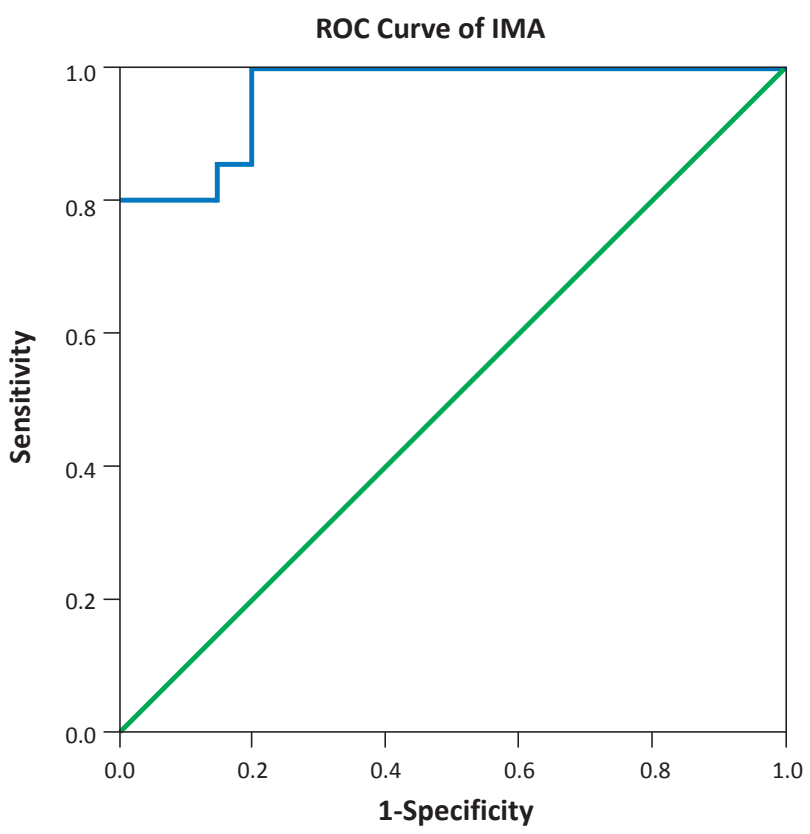

Fig. 1. Receiver operating characteristic curve analysis of ischemiamodified albumin (IMA) levels by group. ROC, receiver operating characteristic curve.

distress syndrome, sepsis, and pneumonia in those babies. However, other studies found no significant relationship between HIE and birth weight. ${ }^{17)}$

Apgar score is still the most achievable and practical to do in the delivery room. The 5-minute Apgar score is still a valid and fast index for estimating the efficacy of resuscitative efforts to the infant. Trevisanuto et al. ${ }^{18)}$ found that 5 minutes Apgar score $<5$ was a significant risk factor for severe HIE. In our study, there was a highly significant difference in Apgar score at 1 and 5 minutes between the 2 studied groups. Other studies consistently showed that low Apgar scores at 5 minutes are associated with greater risk of occurrence of $\mathrm{HIE}{ }^{15,17,18)}$

Our study demonstrates no significant association between gender and brain insult, this is in matching with previous studies. ${ }^{19)}$ However, male sex predominance among newborns with perinatal asphyxia was evident in many studies. ${ }^{20)}$ This finding was explained by Greenough et al. ${ }^{21)}$ who found that male fetuses have lower catecholamine levels than their female peers and so are less protected against intrapartum hypoxia and its consequences. Furthermore, Lang and McCullough ${ }^{22)}$ reported that females are more protected from hypoxia and ischemic neuronal cell death than males due to the neuroprotective effects of estrogen (17 $\beta$ estradiol or E2) against ischemic insults. In both groups, there was no statistically significant difference between the mean cord blood IMA levels in neonates from cesarean sections compared to those from vaginal deliveries. This has helped to remove the effect of different modes of deliveries on oxidative stress.

The mortality rate in this study was 12 cases ( 4 cases in stage II, 8 cases in stage III) from 60 asphyxiated neonates with a percent equal to $20 \%$ which is near to the results of Campbell et al. ${ }^{23)}$ 
who reported that asphyxia as a secondary cause of neonatal mortality in Egypt (18\%) after prematurity as a primary cause.

Our result revealed a highly significant relation between prolonged hospital stay and severity of HIE in asphyxiated neonates, which runs with a previous study. ${ }^{24)}$ However, our findings were not consistent with other studies which determined that the short-term outcomes of infants depend on perinatal academia, 5-minute Apgar score $<4$, cord arterial $\mathrm{pH}<7.1$, and base deficit $>20$ and did not depend on the duration of hospital stay. ${ }^{12,25)}$

We found that the hemoglobin level was significantly lower in the asphyxiated group than controls. It agrees with Caliskan et al. ${ }^{26}$ and Brucknerová et al. ${ }^{27)}$ Who assumed that anemia was caused by increase of oxidative stress during prenatal and direct postnatal period, as well as by hemorrhage or by blood redistribution (adaptive mechanisms which assist keep up ideal blood supply to the most sensitive organs such as central nervous system, heart). In the present study, CRP was higher in the asphyxiated group than controls. This agrees with Shang et al. ${ }^{28)}$ indicating the presence of an acute inflammatory response in the patients. We did not find a significant difference in the CRP level between the 3 stages of the asphyxiated group but Shang et al. ${ }^{28)}$ found a correlation between the elevation of CRP and the severity of the disease. Thus, patients with greater inflammatory responses suffered from a severe progression of the disease. Regarding arterial blood gases ( $\mathrm{ABG}$ ) values, the mean $\mathrm{pH}$ values and $\mathrm{PO}_{2}$ were significantly lower in HIE infants than the control group while the mean $\mathrm{pCO}_{2}$ was significantly elevated in HIE infants than the control group. Acidosis was attributed to increased fetal carbon dioxide level and anaerobic metabolism that runs with other studies. ${ }^{18,29)}$ But, our study disagrees with Khan et al. ${ }^{30)}$ who did not find a significant difference between control and asphyxiated group regarding ABG values.

IMA determination is a biochemical test for HIE diagnosis that provides results immediately after asphyxia even at birth, as reported in previous studies. ${ }^{31,32)}$ Serum IMA sample was taken from the cord before initiating any HIE treatment.

Our study found that the cord blood IMA levels were significantly elevated (nearly double elevation) in hypoxic than healthy full-term neonates, this runs with other studies that found a significant increase in IMA levels in perinatal asphyxia than the control subjects. Firstly, Gugliucci et al. ${ }^{32)}$ who found that the cord blood IMA levels in neonates from complicated deliveries are $50 \%$ higher than in neonates from uneventful deliveries $(P<0.05)$ while their albumin values are not significantly different and hypothesized that decreased blood flow in complicated deliveries results in insufficient oxygenation, anaerobic metabolism, localized acidosis and may increase IMA and concluded that cord blood IMA levels could be used as a marker for fetal ischemia in complicated pregnancies. Also, Kar et al. ${ }^{33)}$ found a significant elevation of IMA in cord blood of babies born to preeclamptic mothers. Secondly, Iacovidou et al. ${ }^{34)}$ who found that the reactive oxygen species, produced during ischemia/ reperfusion, can create the highly reactive hydroxyl radical caus- ing a site-specific modification of albumin and producing IMA. Thirdly, Kumral et al. $\left.{ }^{35}\right)$ Who found that newborns with perinatal asphyxia had signicantly higher cord blood IMA levels than the non-asphyxiated ones $(P=0.015)$ and also, Biswas et al. ${ }^{17)}$ who revealed that cord blood IMA levels were higher in asphyxiated newborns compared to controls. All these studies concluded that elevated cord blood IMA levels may be accepted as a useful marker in perinatal asphyxia but they did not study its role in predicting the severity of the injury. ${ }^{17,34-36)}$

We found a highly significant difference between the 3 stages of the HIE group regarding the cord blood IMA levels which was attributed to increased oxidative stress caused by ischemiareperfusion injury or other mechanisms related to a primary decrease of coronary blood flow and muscle damage. The results of the present study supported the usefulness of IMA level for diagnosing HIE as its advantages are easy blood sample taking (a small quantity of blood can be obtained from the cord before clamping in the full-term neonate presents with asphyxia event), it is easy to determine, it is of low cost. These advantages favor the view that IMA is a biochemical marker that can demonstrate the presence of neuronal change early, so help in early diagnosis without waiting for clinical manifestations.

Our study demonstrated an inverse correlation between IMA and Apgar score at 1, 5 minutes as its value increases with decreased Apgar score in asphyxiated babies. This agreed with other studies. ${ }^{1734)}$ These data are significant for supporting early diagnosis and having the capacity to start therapeutic measures for preventing mortality and long-term neurological sequelae. Also, we found a significant positive correlation between IMA level and birth weight, hospital stay in the asphyxiated group. In comparison to the control group, where there was no statistically significant correlation between IMA and all these variables. Regarding laboratory findings, our study revealed inverse correlations between IMA and $\mathrm{pH}, \mathrm{PO}_{2}$. Also, we found significant positive correlations between IMA and CRP, $\mathrm{PCO}_{2}$ in the asphyxiated group. In comparison to the control group, where there was no significant correlation between IMA and all variables.

Regarding validity of IMA as a diagnostic marker of HIE, this study shows that the best cutoff value of serum IMA is $197.6 \mathrm{pmol} / \mathrm{mL}$ with sensitivity $84.5 \%$, specificity $86 \%$, PPV $82.8 \%$, NPV $88.3 \%$, which is superior to other markers such as nucleated red blood cells which showed a sensitivity of $83.4 \%$ and specificity of $73.5 \%$.

The limitation of the present study was the relatively small number of patients participating in the study and we did not evaluate the role of IMA as a prognostic marker after the administration of neuroprotective therapies.

We concluded that IMA can be reliable markers not only for early diagnosis of neonatal HIE but also for predicting the severity of the injury. Therefore, it can protect asphyxiated neonates from permanent neurological damage and mortality by early administration of neuroprotective therapies. 


\section{Conflicts of interest}

No potential conflict of interest relevant to this article was reported.

\section{Acknowledgments}

The authors thank the staff of Neonatology unit in Zagazig University Children's Hospital for their collaboration as well as the parents of children's who accepted to participate in the study.

\section{References}

1. Distefano G, Praticò AD. Actualities on molecular pathogenesis and repairing processes of cerebral damage in perinatal hypoxic-ischemic encephalopathy. Ital J Pediatr 2010;36:63. https://doi.org/10.1186/18247288-36-63.

2. Vannucci SJ, Hagberg H. Hypoxia-ischemia in the immature brain. J Exp Biol 2004;207(Pt 18):3149-54.

3. Florio P, Abella R, Marinoni E, Di Iorio R, Li Volti G, Galvano F, et al. Biochemical markers of perinatal brain damage. Front Biosci (Schol Ed) 2010;2:47-72.

4. Bar-Or D, Lau E, Winkler JV. A novel assay for cobalt-albumin binding and its potential as a marker for myocardial ischemia-a preliminary report. J Emerg Med 2000;19:311-5.

5. Shen XL, Lin CJ, Han LL, Lin L, Pan L, Pu XD. Assessment of ischemiamodified albumin levels for emergency room diagnosis of acute coronary syndrome. Int J Cardiol 2011;149:296-8.

6. Sbarouni E, Georgiadou P, Kremastinos DT, Voudris V. Ischemia modified albumin: is this marker of ischemia ready for prime time use? Hellenic J Cardiol 2008;15:260-6.

7. Hekimoğlu B, Yakup Aslan Y, Mutlu M, Menteşe A, Karahan SC. Predictive values of Ischemia modified albumin in neonatal sepsis. Turk J Biochem 2017;3:245-50.

8. Kahveci H, Tayman C, Laoğlu F, Celik HT, Kavas N, Kılıç Ö et al. Serum ischemia-modified albumin in preterm babies with respiratory distress syndrome. Indian J Clin Biochem 2016;31:38-42.

9. Özdemir ÖM, Özdemir E, Enli Y, Öztekin Ö, Ergin H. Ischemia-modified albumin in preterm infants born to mothers with pre-eclampsia. Pediatr Int 2018;60:553-9.

10. Florio P, Frigiola A, Battista R, Abdalla Ael H, Gazzolo D, Galleri L, et al. Activin A in asphyxiated full term newborns with hypoxic ischemic encephalopathy. Front Biosci (Elite Ed) 2010;2:36-42.

11. Levene MI, Kornberg J, Williams THC. The incidence and severity of post-asphyxial encephalopathy in full-term infants. Early Hum Dev 1985;11:21-8.

12. Finer NN, Robertson CM, Richards RT, Pinnell LE, Peters KL. Hypoxicischemic encephalopathy in term neonates: perinatal factors and outcome. J Pediatr 1981;98:112-7.

13. Ballard JL, Khoury JC, Wedig K, Wang L, Eilers-Walsman BL, Lipp R. New Ballard Score expanded to include extremely premature infants. J Pediatr 1991;119:417-23.

14. Sarnat HB, Sarnat MS. Neonatal encephalopathy following fetal distress. A clinical and electroencephalographic study. Arch Neurol 1976;33:696705.

15. Fatrakul S, Praisuwanna P, Thaitumyanon P. Risk factors for hypoxic. Ischemic encephalopathy in asphyxiated newborn infants. J Med Assoc Thai 2006;89:322-8.

16. Lee AC, Kozuki N, Blencowe H, Vos T, Adil Bahalim A, Gary L. et al. Intrapartum-related neonatal encephalopathy incidence and impairment at regional and global levels for 2010 with trends from 1990. Pediatr Res 2013;74 Suppl 1:50-72.
17. Biswas S, Sarkar P, Chakraborty I, Ghosh S, Halder RC. Study of cord blood ischemia-modified albumin levels in the evaluation of birth asphyxia. J Invest Biochem 2014;3:46-50.

18. Trevisanuto D, Picco G, Golin R, Doglioni N, Altinier S, Zaninotto M, et al. Cardiac troponin I in asphyxiated neonates. Biol Neonate 2006;89: 190-3.

19. Kirimi E, Peker E, Tuncer O, Yapicioglu H, Narli N, Satar M. Increased serum malondialdehyde level in neonates with hypoxic-ischaemicencephalopathy: prediction of disease severity. J Int Med Res 2010;38:2206.

20. Hill CA, Fitch RH. Sex differences in mechanisms and outcome of neonatal hypoxia-ischemia in rodent models: implications for sex-specific neuroprotection in clinical neonatal practice. Neurol Res Int 2012;2012: 867531.

21. Greenough A, Lagercrantz H, Pool J, Dahlin F. Plasma catecholamine levels in preterm infants. Effect of birth asphyxia and Apgar score. Acta Paediatr Scand 1987;76:54-9.

22. Lang JT, McCullough LD. Pathways to ischemic neuronal cell death: are sex differences relevant? J Trans Med 2008;6:33.

23. Campbell O, Gipson R, el-Mohandes A, Issa AH, Matta N, Mansour E, et al. The Egypt National Perinatal/Neonatal Mortality Study 2000. J Perinatol 2004;24:284-9.

24. Carli G, Reiger I, Evans N. One-year neurodevelopmental outcome after moderate newborn hypoxic ischaemic encephalopathy J Paediatr Child Health 2004;40:217-20.

25. Toh VC. Early predictors of adverse outcome in term infants with postasphyxial hypoxic ischaemic encephalpathy. Acta Paediatr 2000;89:3437.

26. Caliskan E, Doger E, Cakiroglu Y, Duman C, Turker G, Yucesoy I. Cord blood cardiac troponin I and creatine kinase MB levels in poor neonatal outcomes. J Turkish German Gynecol Assoc 2006;7:98-102.

27. Brucknerová I, Ujházy E, Dubovický M, Mach M. Early assessment of the severity of asphyxia in term newborns using parameters of blood count. Interdiscip Toxicol 2008;1(3-4):211-3.

28. Shang Y, Mu L, Guo X, Li Y, Wang L, Yang W, et al. Clinical significance of interleukin- 6 , tumor necrosis factor- $\alpha$ and high-sensitivity C-reactive protein in neonates with hypoxic-ischemic encephalopathy. Exp Ther Med 2014;8:1259-62.

29. Schmidt JW, Walsh WF. Hypoxic-ischemic encephalopathy in preterm infants. J Neonatal Perinatal Med 2010;3:277-84.

30. Khan KS, Bachmann LM, Ter Riet G. Systematic reviews with individual patient data meta-analysis to evaluate diagnostic tests. Eur J Obstet Gynecol Reprod Biol 2003;108:121-5.

31. Gazzolo D, Abella R, Marinoni E, Iorio R, Li Volti G, Galvano F, et al. Circulating biochemical markers of brain damage in infants complicated by ischemia reperfusion injury. Cardiovasc Hematol Agents Med Chem 2009;7:108-26.

32. Gugliucci A, Hermo R, Monroy C, Numaguchi M, Kimura S. Ischemiamodified albumin levels in cord blood: a case-control study in uncom. plicated and complicated deliveries. Clin Chim Acta 2005;362:155-60.

33. Kar K, Biswas M, Das S, BiswasT, Biswas J, Sarkar C. Cord blood ischemia modified albumin may predict the oxidative stress in newborns, a prospective study in Kolkata. Intern J of Health Sci Res 2015;5:178-83.

34. Iacovidou N, Briana DD, Boutsikou M, Liosi S, Baka S, Boutsikou T, et al. Cord blood ischemia-modified albumin levels in normal and intrauterine growth restricted pregnancies. Mediators Inflamm 2008;2008:523081.

35. Kumral A, Okyay E, Guclu S, Gencpinar P, Islekel GH, Oguz SS, et al. Cord blood ischemia-modified albumin: is it associated with abnormal Doppler findings in complicated pregnancies and predictive of perinatal asphyxia? J Obstet Gynaecol Res 2013;39:663-71.

36. Dursun A, Okumus N, Zenciroglu A. Ischemia-modified albumin (IMA): could it be useful to predict perinatal asphyxia? J Matern Fetal Neonatal Med 2012;25:2401-5. 